\title{
Wire Density Driven Global Routing for CMP Variation and Timing
}

\author{
Minsik Cho, David Z. Pan \\ ECE Dept. Univ. of Texas at Austin \\ Austin, TX 78712 \\ \{mcho,dpan\}@ece.utexas.edu
}

\author{
Hua Xiang, Ruchir Puri \\ IBM T. J. Watson Research Center \\ Yorktown Heights, NY 10598 \\ \{huaxiang,ruchir\}@us.ibm.com
}

\begin{abstract}
In this paper, we propose the first wire density driven global routing that considers CMP variation and timing. To enable CMP awareness during global routing, we propose a compact predictive CMP model with dummy fill, and validate it with extensive industry data. While wire density has some correlation and similarity to the conventional congestion metric, they are indeed different in the global routing context. Therefore, wire density rather than congestion should be a unified metric to improve both CMP variation and timing. The proposed wire density driven global routing is implemented in a congestion-driven global router [5] for CMP and timing optimization. The new global router utilizes several novel techniques to reduce the wire density of CMP and timing hotspots. Our experimental results are very encouraging. The proposed algorithm improves CMP variation and timing by over $7 \%$ with negligible overhead in wirelength and even slightly better routability, compared to the pure congestion-driven global router [5].
\end{abstract}

\section{Categories and Subject Descriptors}

B.7.2 [Hardware, Integrated Circuit]: Design Aids General Terms

Algorithms, Design, Performance

\section{Keywords}

VLSI, Global Routing, Performance, Manufacturability

\section{INTRODUCTION}

Aggressive technology scaling has led to much higher resistance and larger coupling capacitance on interconnect. According to ITRS roadmap [16], copper dishing/erosion after Chemical-Mechanical Polishing (CMP) and scattering effect may increase resistance significantly $[7,15,29]$. Also, coupling capacitance between wires becomes dominant over ground and fringing capacitance at $0.18 \mu \mathrm{m}$ technology (over $60 \%$ of the total capacitance), and increases

This work is supported in part by SRC, IBM Faculty Award, Fujitsu, and equipment donations from Intel.

Permission to make digital or hard copies of all or part of this work for personal or classroom use is granted without fee provided that copies are not made or distributed for profit or commercial advantage and that copies bear this notice and the full citation on the first page. To copy otherwise, to republish, to post on servers or to redistribute to lists, requires prior specific permission and/or a fee.

ICCAD 2006, November 5-9, 2006, San Jose, CA, USA.

Copyright 2006 ACM 1-59593-389-1/06/0011 ...\$5.00. rapidly with higher wire aspect ratio of the advanced technologies $[2,26,27]$. Therefore, interconnect delay will suffer from the increased resistance and coupling capacitance more seriously in the future.

Meanwhile, manufacturability and yield are becoming everserious concerns at $90 \mathrm{~nm}$ node and below, and shown to be heavily affected by design patterns [21]. Especially, topography (thickness) variation after CMP is shown to be systematically determined by wire density distribution [19,21,29]. Even after CMP, intra-chip topography variation can still be on the order of $20-40 \%[9,21]$. Such topography variation leads to not only significant performance degradation due to increased wire resistance and capacitances, but also acute manufacturing issues like etching and printability $[7,9,21,23]$.

The main reason for the above problems is wire density distribution. Higher wire density usually leads to copper thickness reduction due to erosion after CMP [19, 29], making resistance worse. Also, the reduced copper thickness after CMP can worsen the scattering effect, further increasing resistance [15]. Meanwhile, a region with higher wire density tends to have less spacing between wires, thus significantly increasing coupling capacitance between them. For a lower wire density region, dummy fill is performed before CMP to make density distribution more uniform, expecting less topography variation after CMP. However, dummy fill is usually applied in the post-design stage, and has limitations due to strict rules (such as fill size, patten, spacing to other features) which intend to minimize the disturbance on the existing design. Thus, non-uniform distribution of density still exists even after the dummy fill, creating topography variation which wastes the minuscule depth of focus [6].

The global routing, as its name implies, is the routing stage that plans the approximate routing path of each net to reduce the complexity of routing task and guide the detailed router [14]. Thus, it has significant impact on wirelength, congestion/routability and wire density distribution, hence CMP variation and timing $[7,13,14,25]$. The global routing should be an ideal stage for the first order to improve CMP variation as well as timing by optimizing wire density distribution $[4,26]$. Even though there have been significant amount of work in global routing about crosstalk $[4,10,17,18$, $22,24,30]$, timing $[4,13,26,28]$ and congestion/routability [1, $5,8]$, none of them has worked on the optimization of wire density distribution for CMP variation and timing.

In this paper, we propose a new paradigm of wire density driven global routing to improve CMP variation and timing. To our best knowledge, this is the first work that takes 
wire density distribution into account to enhance both CMP variation and timing, while considering congestion and wirelength. Essentially, the proposed algorithm minimizes the wire density around timing critical nets to enhance timing, and the wire density of dense global routing cells to reduce topography variation after CMP. The major contributions of this paper include the following.

- We present a simple predictive CMP model verified with industrial cases for the first time. We also show that only few regions of a chip are responsible for most of topography variation after CMP. This means that selective optimization of wire density distribution can enhance the uniformity of topography.

- We use the wire density to guide global routing for CMP variation and timing. We show that wire density and congestion are not linearly proportional to each other. This implies that just congestion-driven global routing cannot address the wire density issue efficiently. Furthermore, we observe that wire delay is near linearly proportional to wire density in global routing even with CMP effect (dishing/erosion and dummy fill) $[9,20,29]$ and scattering effect [15] taken into consideration.

- We propose several novel wire density driven routing techniques for CMP variation and timing optimizations, including minimum pin density routing, timing sensitivity map and CMP aware wire density distribution. Each technique helps global router to reduce the wire density around timing critical nets, while minimizing topography variation after CMP.

Our approach achieves better timing and less topography variation than the state-of-the-art academic congestiondriven router [5] with negligible overhead on wirelength, and even better congestion/routability.

The rest of the paper is organized as follows. In Section 2, preliminaries are described. A predictive CMP model is presented, and timing impact with wire density distribution is analyzed in Section 3. In Section 4, a wire density driven global routing is proposed. Experimental results are discussed in Section 5. Section 6 concludes this paper.

\section{PRELIMINARIES}

\subsection{Global Routing Model}

The global routing problem can be modelled as a grid graph $G(V, E)$, where each vertex $v_{i}$ represents a rectangular region of the chip, so called a global routing cell (G-cell), and each edge $e_{i j}$ represents the boundary between $v_{i}$ and $v_{j}$ with a given maximum routing resource $r_{i j}$. A global routing is to find paths that connect the pins inside the Gcells through $G(V, E)$ for each net.

\subsection{Global Routing Metrics}

The key task of global router is to maximize (minimize) the routability (congestion) for successful detailed routing [25]. In addition, timing, manufacturability, and wirelength are other important goals of global router.

- Routability can be estimated by the number of overflows which indicates that routing demand locally exceeds the available routing capacity [25] [17]. Formal definition of overflow is in [17].
Table 1: The notations in this paper.

\begin{tabular}{|c|c|}
\hline$v_{i}$ & vertex / global routing cell $i$ \\
\hline$w_{i}$ & wire density of $v_{i}$ \\
\hline$d_{i}$ & dummy density of $v_{i}$ \\
\hline$m_{i}$ & metal density of $v_{i}$ \\
\hline$t_{i}$ & copper thickness of $v_{i}$ \\
\hline$l_{i}$ & width / height of $v_{i}$ \\
\hline$n_{i}$ & number of signal wires in $v_{i}$ \\
\hline$p_{i}$ & number of wide wires in $v_{i}$ \\
\hline$s_{i}$ & average spacing between wires in $v_{i}$ \\
\hline$t s_{i}$ & timing sensitivity of $v_{i}$ \\
\hline$e_{i j}$ & edge between $v_{i}$ and $v_{j}$ \\
\hline$r_{i j}$ & maximum routing resource of $e_{i j}$ \\
\hline$c_{i j}$ & available routing resource of $e_{i j}$ \\
\hline$g_{i j}$ & congestion of $e_{i j}$ \\
\hline
\end{tabular}

- Timing is a significant design objective, as interconnect which governs the overall system performance is mainly determined by global routing $[13,26]$.

- Manufacturability is no longer an optional objective in deep submicron region. In global routing, CMP variation which is a macro systematic variation due to wire density distribution can be optimized, as global router plans approximate routing path for each net [14].

- Wirelength is an important metric for placement as well as routing. But, it is less concern for global routing, as routing all wires with shortest path algorithms will result in minimum or near-minimum wirelength [25].

\section{PREDICTIVE CMP MODEL AND TIM- ING IMPACT WITH WIRE DENSITY}

In this section, we propose a predictive CMP model for the first time. It is followed by the study of the timing impact of wire density under CMP (topography variation and dummy fill) [7] and scattering effect [15]. Then, we show that congestion driven routing cannot replace wire density driven routing, even though they are closely related.

\subsection{Wire Density and Predictive CMP Model}

Topography variation (thickness variation) after CMP is determined by underlying metal density which includes both wires and dummies. As dummy fill in turn depends on wire density, the required dummy density and the copper $(\mathrm{Cu})$ thickness can be predicted from a given wire density.

In Fig. 1, normalized $\mathrm{Cu}$ thickness change by metal density is shown based on three industrial designs. For a given G-cell $v_{i}$ with a metal density $m_{i}$, we discover the $\mathrm{Cu}$ thickness of $v_{i}, t_{i}$ can be expressed as follows:

$$
t_{i}=\alpha\left(1-\frac{m_{i}^{2}}{\beta}\right) \quad\left(0.2 \leq m_{i} \leq 0.8\right)
$$

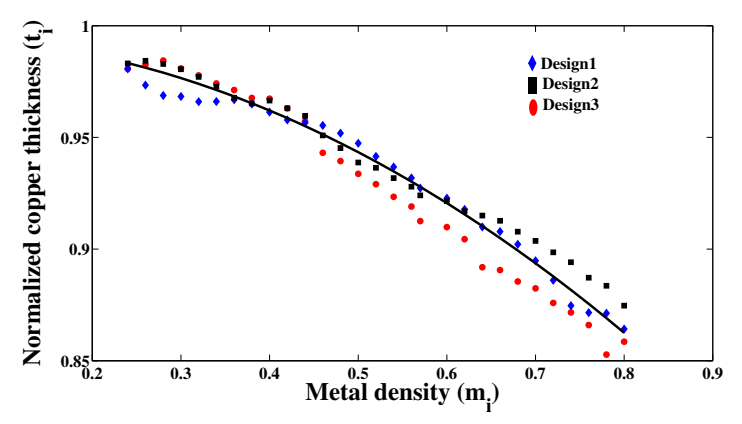

Figure 1: Normalized Cu thickness by metal density 


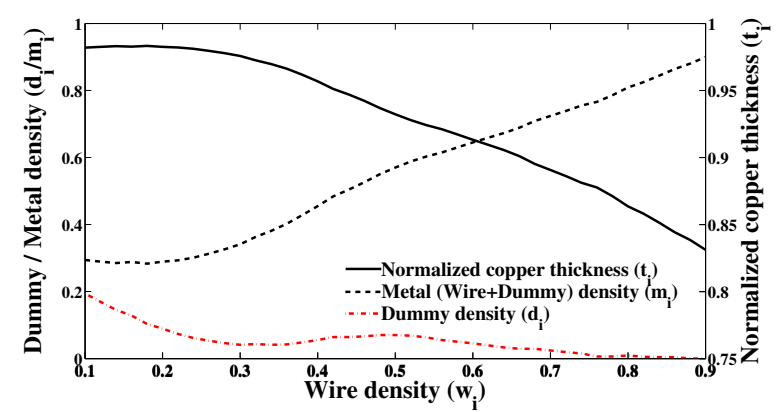

Figure 2: Predictive CMP model

where $\alpha$ and $\beta$ are technology dependent constants. Eq. (1) requires the metal density $m_{i}$ as an input which is essentially the summation of the wire density $w_{i}$ and the dummy density $d_{i}$ in a global routing cell $v_{i}$. Fig. 2 shows the required dummy density and the predicted $\mathrm{Cu}$ thickness with respect to wire density. For a given $v_{i}, d_{i}$ can be looked up with $w_{i}$ using Fig. 2, and then $m_{i}$ can be obtained by adding $w_{i}$ and $d_{i}$. Finally, the calculated $m_{i}$ can be fed into Eq. (1) to predict the $\mathrm{Cu}$ thickness $t_{i}$. This predictive model is verified with a commercial CMP simulator [12] and industry test cases.

\subsection{Wire Density and Timing}

For two adjacent wires, each has two capacitance components, coupling capacitance $C_{c}$ between itself and the other wire, and ground capacitance $C_{g}$ between itself and ground. Bakoglu [3] shows that wire delay on a distributed $R C$ network contains the following delay component, $D$ :

$$
D \propto\left(R_{d}+r \cdot l\right)\left(C_{g}+C_{c}\right)=\left(R_{d}+\frac{\rho \cdot l}{w \cdot t}\right)\left(\frac{\epsilon \cdot l \cdot w}{h}+\frac{\epsilon \cdot l \cdot t}{s}\right)
$$

where $R_{d}$ is the driver resistance, $\rho$ is the resistivity of the wire, $\epsilon$ is the insulator dielectric constant, $w, t$ and $h$ are the wire width, thickness and distance from the substrate, respectively. And, $l$ and $s$ are the length and spacing between two coupled wires, respectively.

In Eq. (2), $h$ is a constant and $w$ can be assumed to be a constant as well (we do not consider the width variation effect in this paper as it is relatively small for $\mathrm{Cu}$ process). Without loss of generality, we just use the nominal wire width. For a given G-cell $v_{i}, t_{i}$ is essentially a function of $w_{i}\left(m_{i}\right.$ depends on $w_{i}$ as in Fig. 2), and can be denoted as $t_{i}\left(w_{i}\right)$. If even spacing between wires and square G-cell are assumed, spacing between wires $s_{i}$ becomes a function of $w_{i}$ :

$$
s_{i}\left(w_{i}\right)=l_{i} \cdot \frac{1-w_{i}}{n_{i}+p_{i}} \propto \frac{1}{w_{i}}-1 \quad\left(0<w_{i} \leq 1\right)
$$

Also, we can obtain the increased total capacitance due to dummy fill (dummy increases coupling capacitance) as a function of $w_{i}$ based on [20]:

$$
f\left(w_{i}\right)=1+\gamma \log \left(\frac{s_{i}\left(w_{i}\right)}{\delta}\right)
$$

where $\gamma$ and $\delta$ are technology dependent constants. Note that $f\left(w_{i}\right)$ is typically less than 1.1, and saturates quickly. Due to scattering effect under a given temperature, $\rho$ is a function of $t_{i}$. Thus, it is also a function of $w_{i}$ (see Eq. (1)), and can be denoted as $\rho\left(w_{i}\right)$ [15]. Finally, the component of the wire delay in $v_{i}$ can be rewritten as a function of $w_{i}$ based on Eq. (2):

$$
\begin{gathered}
R\left(w_{i}\right)=\frac{\rho\left(w_{i}\right) \cdot l_{i}}{t_{i}\left(w_{i}\right) \cdot W_{n o m}} \\
C\left(w_{i}\right)=\epsilon \cdot f\left(w_{i}\right)\left(\frac{l_{i} \cdot W_{n o m}}{h}+\frac{l_{i} \cdot t_{i}\left(w_{i}\right)}{s_{i}\left(w_{i}\right)}\right) \\
D\left(w_{i}\right) \propto\left(R_{d}+R\left(w_{i}\right)\right) C\left(w_{i}\right) \propto w_{i}
\end{gathered}
$$

We observe the following with $65 \mathrm{~nm}$ technology node:

- Lower wire density shows less resistance (less erosion after CMP) as well as less capacitance (more spacing between wires).

- Resistance variation dependence on wire density (computed post dummy fill and CMP estimation) is relatively smaller than capacitance variation.

- $D\left(w_{i}\right)$ is near linearly proportional to $w_{i}$ in the region where most of wire densities exist (see Eq. (3) and (6)).

\subsection{Wire Density and Congestion}

While congestion/routability has been the main concern in global routing, wire density distribution is an emerging important objective due to CMP variation and timing as shown in Section 3.1 and 3.2. Although wire density is closely related to congestion, congestion driven global routing cannot address wire density issues directly and effectively, because wire density is computed for the wires inside a global routing cell, while congestion is computed for the wires crossing the edges of a global routing cell.

Fig. 3 shows a G-cell with edge $A$ and $B$. When there is a wide wire such as power/ground as in Fig. 3 (a), density is not a half of congestion. Even though Fig. 3 (a) and (b) have the same density, Fig. 3 (b) is less congested. As wire density and congestion can be significantly different, wire density cannot be replaced with congestion; as a unified metric, wire density should be directly addressed in global routing for the optimization of CMP variation and timing.

\section{WIRE DENSITY DRIVEN GLOBAL ROUTING FOR CMP AND TIMING}

In this section, we propose our wire density driven global routing algorithm which essentially minimizes the delay of timing critical nets by decreasing the wire density around such nets, and improves the topography variation after CMP by decreasing the wire density of dense G-cells selectively. Our approach consists of four ideas which can be easily integrated with any existing global router [1,5,8,17]; minimum pin density routing in Section 4.1, timing sensitivity map in Section 4.2, selective wire density optimization for CMP awareness in Section 4.3, and wire density driven maze routing in Section 4.4 .

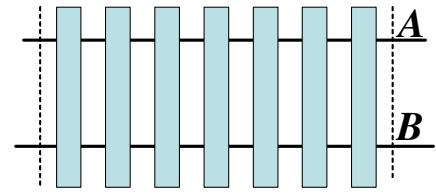

(a) den $=50 \%, g_{A}=g_{B}=100 \%$

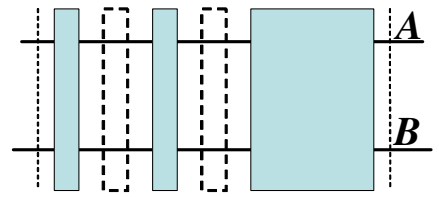

(b) den $=50 \%, g_{A}=g_{B}=71 \%$
Figure 3: Difference between congestion and density 


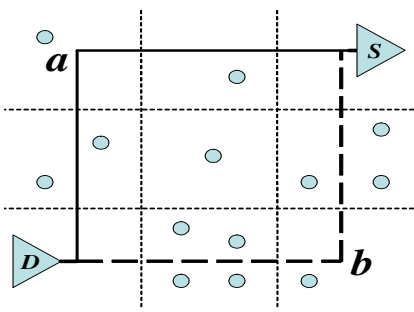

(a) two possible paths for $D-S$

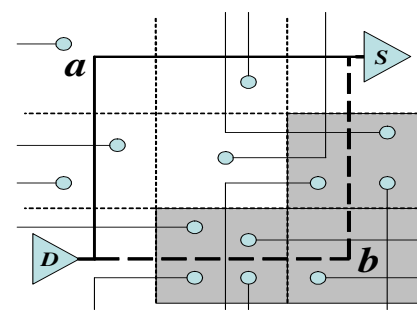

(b) path $b$ with higher density
Figure 4: Example of minimum pin density routing

\subsection{Minimum Pin Density Routing}

In practice, timing critical nets are commonly routed before other nets for the minimum length. As there can be multiple paths with the minimum length, a router can choose any of those based on its policy. However, we find that a path with the minimum pin density is better for the wire density distribution. In Fig. 4 , a net $D-S$ to be routed is shown with a pin distribution. If only L-shape routing is allowed, we can have two possible paths, $a$ and $b$ with the same length but different pin densities. As the existence of a pin means a connection to other pins, a path with higher pin density like $b$ tends to have higher wire density eventually as in Fig. 4 (b), resulting in higher resistance and larger coupling capacitance.

More importantly, the path with higher pin density cannot get much benefit from the wire density optimization, as there is little room for improvement (it is destined to have high wire density from the beginning). Note only L/Z-shape routing is considered in the minimum pin density routing to minimize the number of vias in our implementation.

\subsection{Timing Sensitivity Map Construction}

As Elmore delay can be expressed as the summation of $R C$ product where $C$ is the downstream capacitance seen by $R$ in the distributed $R C$ network, a capacitance close to the sink has more impact on timing than one close to the driver. Also, the capacitance of a G-cell where more timing critical nets are passing has more impact on timing. Thus, we can compute the sensitivity of each G-cell to timing, and construct the timing sensitivity map. As resistance is relatively insensitive to wire density variation (see Section 3.2), we consider only capacitance in constructing the timing sensitivity map. Fig. 5 illustrates how to construct timing sensitivity map. Given a net $D_{1}-S_{1}-S_{2}$ as in Fig. 5 (a), if nominal resistance $R$ is assumed for each G-cell (see Section 3.2), a part of Elmore delay from $D_{1}$ to $S_{1}$ can be written as follows:

$$
\begin{aligned}
\text { Delay } & \propto R C\left(w_{a}\right)+2 R C\left(w_{b}\right)+4 R C\left(w_{f}\right) \\
& +3\left\{R C\left(w_{c}\right)+R C\left(w_{d}\right)+R C\left(w_{e}\right)\right\}
\end{aligned}
$$

As mentioned in Section 3.2, RC wire delay in a G-cell $v_{i}$ is near linearly proportional to $w_{i}$. Thus, the above delay equation can be further simplified as follows:

$$
\text { Delay } \propto w_{a}+2 w_{b}+4 w_{f}+3\left\{w_{c}+w_{d}+w_{e}\right\}
$$

It is clear that each wire segment (thus, each G-cell) has different impact on the timing due to its geometrical location (whether it is close to driver or not). For example, $10 \%$ increase of $w_{f}$ has the same impact as $40 \%$ increase of $w_{a}$

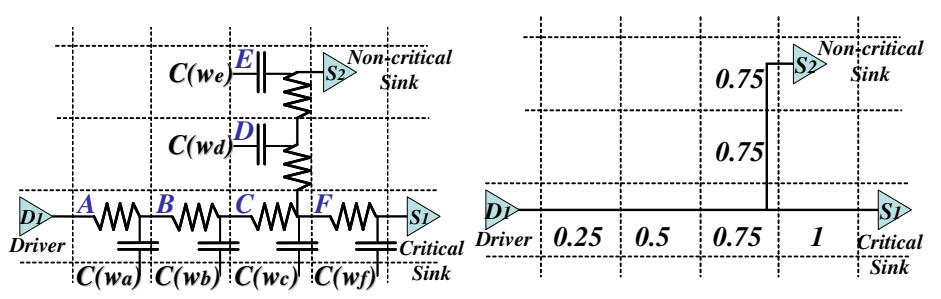

(a) Elmore delay of $D_{1}-S_{1}-S_{2}$

(b) G-cell weights from $D_{1}-S_{1}-S_{2}$
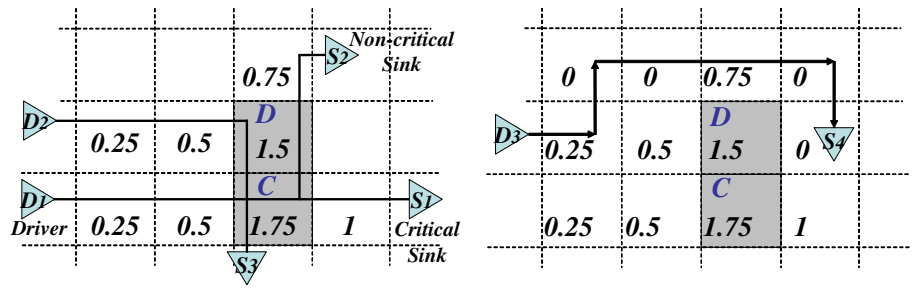

(c) updated weight after $D_{2}-S_{3}$

(d) routed non-critical $D_{3}-S_{4}$

Figure 5: Example of timing sensitivity map construction

on timing. Therefore, each G-cell can have its own time sensitivity to the density change as in Fig. 5 (b). We can apply the same idea to all the timing critical nets which are already routed by the minimum pin density routing, and sum up the timing sensitivity from each net to construct a timing sensitivity map. For example, in Fig. 5 (c), another timing critical net $D_{2}-S_{3}$ is joining the net $D_{1}-S_{1}-S_{2}$ at the G-cell $v_{C}$ and $v_{D}$. As $v_{C}$ has already 0.75 as the timing sensitivity $t s_{C}$, after considering $D_{2}-S_{3}, t s_{C}$ becomes 1.75 due to 1 from $D_{2}-S_{3}$. In the same manner, $t s_{D}$ becomes 1.5 due to another 0.75 from $D_{2}-S_{3}$. With the timing sensitivity of each G-cell, we can make a non-timing critical net $D_{3}-S_{4}$ detour to reduce the wire density of the highly timing sensitive G-cell like $v_{D}$ as in Fig. $5(\mathrm{~d})$. This improves the overall timing by reducing the wire density of more timing sensitive G-cells, while minimizing the overhead in wirelength. Algorithm 1 shows how to construct the timing sensitivity map.

\subsection{CMP Aware Wire Density Distribution}

To improve CMP variation, more uniform metal density distribution should be achieved by increasing density for less dense G-cells or decreasing density for more dense G-cells.

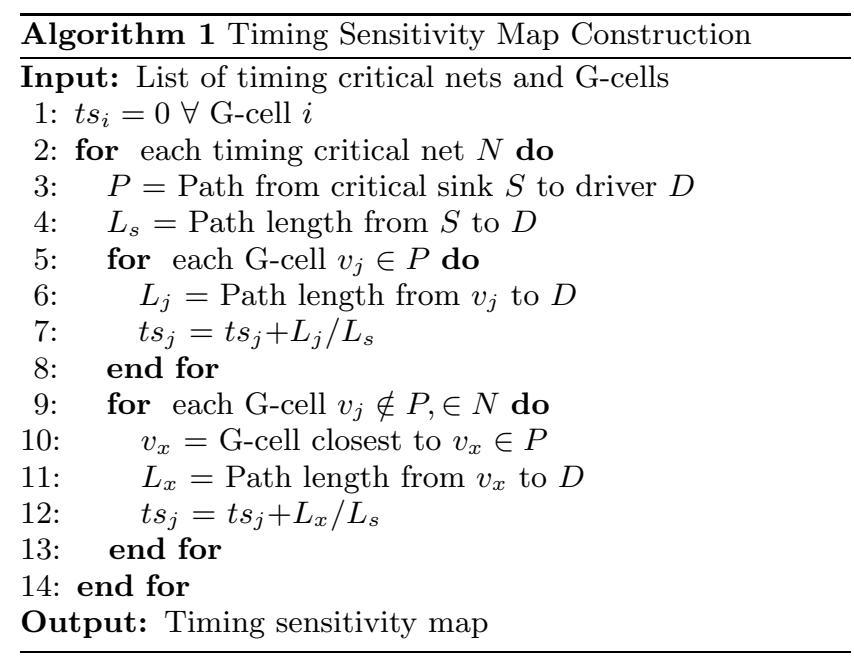




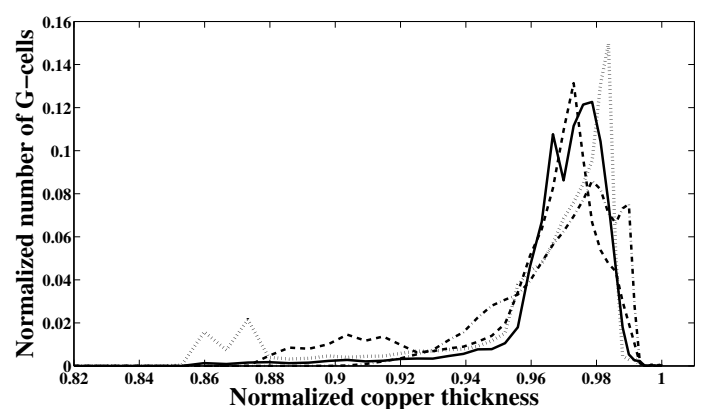

Figure 6: Normalized $\mathrm{Cu}$ thickness distributions of four industrial designs

We find that reducing wire density of the more dense G-cells can be more effective than the other way for two reasons. First, it requires to perturb smaller number of G-cells. Fig. 6 shows $\mathrm{Cu}$ thickness distributions of four industrial cases after CMP. It shows only few G-cells have over $5 \%$ of $\mathrm{Cu}$ loss, and are mainly responsible for topography variation. Thus, optimizing G-cells over a threshold metal density $T_{d}$ can make topography more uniform without disturbing other design objectives. Second, lowering wire density also helps congestion as they are correlated.

\subsection{Wire Density Driven Maze Routing}

The wire density distribution governs both CMP variation and timing. The objective for timing requires lower density for the timing sensitive G-cell, and the objective for CMP variation requires lower density for the G-cells above the threshold of Section 4.3. The wire density driven maze routing is shown in Algorithm 2. Note that $m_{i}$ can be computed from the CMP model in Section 3.1, and Cost are for other design objectives such as congestion. Two parameters, $P$ and $Q$ are introduced to control the trade-off between objectives.

\section{EXPERIMENTAL RESULTS}

We implement the proposed algorithm in $\mathrm{C}++$ by modifying BoxRouter [5], and compare the results with the original BoxRouter. All the experiments are performed on a $2.8 \mathrm{GHz}$ Pentinum-4 Linux machine. The ISPD98 IBM benchmarks are taken from [11], and we modify the horizontal and vertical capacity of each circuit as shown in Table 2. Further, assuming up to $30 \%$ of routing capacity is assigned for wide wires like power/ground, we reduce the routing capacity to get enough number of overflows (to see the impact on congestion/routability), and make the horizontal and the vertical capacity same for easy wire density calculation. Since the original benchmarks do not have any timing informa-

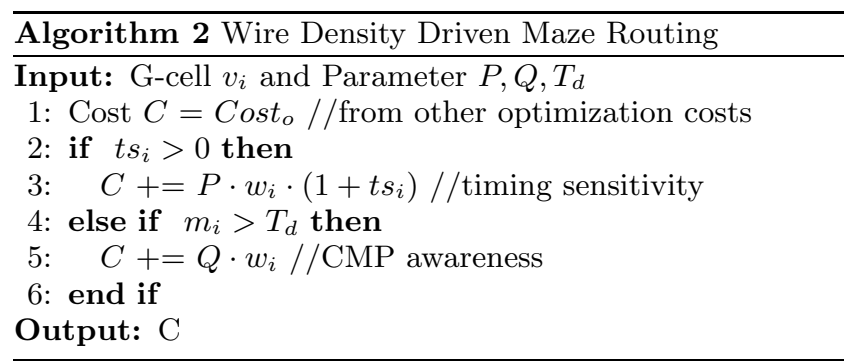

tion, we randomly pick less than $1 \%$ nets which are longer than $200 \mu m$ as optimization targets. HSPICE simulation with $65 \mathrm{~nm}$ technology is performed to estimate timing with CMP variation and scattering effect taken into account. All the timing critical nets are pre-routed in the same minimum length and same topology in any experiment to eliminate the effect of different path lengths and topologies on timing.

Fig. 7 (a) shows total/max delay and wirelength change by the parameter $P$ with $Q=0$. While the wirelength increases as $P$ increases, the delay decreases until $P=1$, and then starts increasing after that. The reason is because too much increase in wirelength due to detouring around timing sensitive G-cells (with higher $P$ value), finally makes overall congestion worse. This provokes the congestion driven objective to push wires back to the timing sensitive G-cells. All the following simulations use $P=1$, as it shows reasonable trade-off. Fig. 7 (b) shows total/max delay, wirelength and topography variation change by the parameter $Q$ with $P=1$. While the total delay and max delay increase with larger $Q$, the topography variation decreases. Wirelength does not change noticeably, proving that our selective wire density optimization for CMP variation is friendly with other design objectives. Although the topography variation can be improved by over $10 \%$ with large $Q$, all the following simulations use $Q=1.3$ for the minimal impact on timing.

Table 2 shows the experimental results and comparison of the proposed algorithm (the modified BoxRouter) with the original BoxRouter. The experiment sets $P=1, Q=1.3$ and $T_{d}=0.33$ of Algorithm 2 to evaluate the optimization for CMP variation and timing together. The average of total delay and max delay are slightly reduced to $7.0 \%$ and $8.0 \%$ respectively, while topography variation after CMP is improved by $7.5 \%$ on average and up to $10.1 \%$. The overhead in CPU time is still only $41 \%$ on average. Also routability is improved, as the number of G-cells with high wire density (which tend to be congested) is reduced by CMP aware wire density distribution. As already shown in Fig. 7 (b), wirelength overhead is quite small, and the improved routability even suppresses the increase in wirelength (less detouring).

\section{CONCLUSION}

In nanometer design, wire density should be a key metric for manufacturability and timing optimization. We present the first wire density driven global routing to improve CMP variation and timing based on the predictive CMP model and several novel techniques. Experimental results are highly encouraging, showing that the proposed algorithm outperforms the state-of-the-art congestion driven global router in terms of CMP variation and timing with negligible overhead in wirelength, and even slightly better routability.

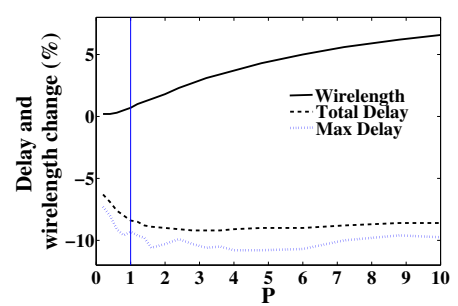

(a) $P$ with $Q=0$

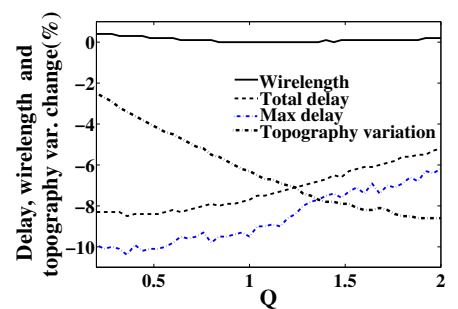

(b) $Q$ with $P=1$
Figure 7: Effectiveness of parameter $P$ and $Q$ 
Table 2: Comparison with BoxRouter for ISPD98 Benchmarks.

\begin{tabular}{|c|c|c|c|c|c|c|c|c|c|c|c|c|c|c|c|c|c|c|c|c|c|c|c|}
\hline \multicolumn{3}{|c|}{ circuit } & \multicolumn{7}{|c|}{ BoxRouter } & \multicolumn{7}{|c|}{ Wire Density Driven BoxRouter } & \multicolumn{7}{|c|}{ Change (\%) } \\
\hline name & $\begin{array}{l}\text { hz. } \\
\text { cap }\end{array}$ & $\begin{array}{l}\text { vt. } \\
\text { cap }\end{array}$ & $\begin{array}{c}\text { t.del } \\
(\mathrm{ns})\end{array}$ & 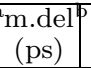 & wlen $^{\mathrm{c}}$ & ovfl & dum ${ }^{\phi}$ & $\begin{array}{c}\mathrm{t} . \mathrm{v}^{\mathrm{f}} \\
(\%)\end{array}$ & $\begin{array}{c}\mathrm{cpu} \\
(\mathrm{sec})\end{array}$ & $\begin{array}{c}\text { t.del } \\
(\mathrm{ns})\end{array}$ & $\begin{array}{c}\text { m.del } \\
(\mathrm{ps})\end{array}$ & wlen & ovfl & dum & $\begin{array}{c}\text { t.v } \\
(\%)\end{array}$ & $\begin{array}{c}\text { cpu } \\
(\mathrm{sec})\end{array}$ & t.del & m.del & wlen & ovfl & dum & t.v & cpu \\
\hline 101 & 18 & 18 & 0.57 & 30.1 & 374 & 4 & 372 & 1.34 & 7.1 & 0.53 & 26.3 & 512 & 5 & 366 & 1.31 & 8.7 & 8.7 & 2.5 & 0.2 & 5.0 & -1.6 & -2.2 & 2.5 \\
\hline 102 & 36 & 36 & 1.85 & 67.6 & 180816 & 25 & 357 & 1.94 & 34.8 & 1.74 & 66.3 & 180399 & 26 & 358 & 1.90 & 44. & -6.0 & -2.0 & -0.2 & 4.0 & 0.3 & -2.1 & 28.4 \\
\hline & 31 & 31 & & 75.7 & 30 & 5 & 389 & 1.73 & & & .4 & 74 & 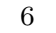 & 39 & 75 & & .0 & & .2 & 20.0 & .0 & .2 & 3.1 \\
\hline a04 & 26 & 26 & 1.61 & 61.4 & 167557 & 99 & 441 & 1.75 & 20.0 & 1.47 & 58.2 & 168307 & 100 & 437 & 1.75 & 26. & -8.4 & -5.2 & 0.4 & 1.0 & -0.9 & 0.0 & 32.0 \\
\hline 105 & 45 & 45 & 3.75 & 252.2 & 426547 & 100 & 542 & 1.78 & 51 & .41 & 219.3 & 432529 & 85 & 539 & 1.84 & 77 . & -9.1 & -13.0 & 1.4 & -15.0 & -0.6 & 3.4 & 52.1 \\
\hline 106 & 35 & 35 & 2.10 & 243.8 & 283836 & 11 & 540 & 1.42 & 33.1 & 1.93 & 228.0 & 286783 & 8 & 541 & 1.46 & 49.7 & -8.3 & -6.5 & 1.0 & -27.3 & 0.2 & 2.8 & 50.2 \\
\hline 07 & 34 & 34 & 1.77 & 0.7 & 372268 & 18 & 867 & 1.67 & & 1.62 & 93.1 & 374954 & 17 & 871 & 1.68 & 63.1 & -8 & -7.5 & 0.7 & -5.6 & 0.5 & 0.6 & 35.7 \\
\hline 08 & 31 & 31 & 2.62 & 191.8 & 420609 & 72 & 770 & 1.72 & 94.2 & 2.36 & 162.4 & 423634 & 62 & 770 & 1.74 & 121.7 & -10.0 & -15.3 & 0.7 & -13.9 & 0.0 & 1.2 & 29.2 \\
\hline & 25 & 25 & 2.32 & 192.8 & 432120 & 75 & 1091 & 1.59 & & 2.13 & 170.4 & 435230 & 70 & 1088 & 1.58 & 88.1 & -8.3 & -11.6 & 0.7 & -6.7 & -0.3 & -0.6 & 28.1 \\
\hline ibm10 & 43 & 43 & 3.21 & 799.0 & 584100 & 33 & 1183 & 1.48 & 90.5 & 2.85 & 711.4 & 589234 & 32 & 1166 & 1.48 & 122.3 & -11.4 & -11.0 & 0.9 & -3.0 & -1.4 & 0.0 & 35.1 \\
\hline & & & & & & & & & & & & & & & $e$ & & -7.0 & -8.0 & 0.0 & -28.6 & -2.2 & -7.5 & 40.9 \\
\hline
\end{tabular}

(a) total delay (b) max delay (c) wirelength (d) overflow (e) the amount of dummy (f) topography variation after dummy fill(std/avg)

\section{REFERENCES}

[1] C. Albrecht. Global Routing by New Approximation Algorithms for Multicommodity Flow. IEEE Trans. on Computer-Aided Design of Integrated Circuits and Systems, 20, May 2001.

[2] R. Arunachaiam, K. rajagopal, and L. T. Pileggi. TACO: Timing Analysis with COupling. In Proc. Design Automation Conf., June 2000.

[3] H. B. Bakoglu. Circuits, Interconnections, and Packaging for VLSI. In Addison-Wesley Publishing Company, 1990.

[4] M. R. Becer, D. Blaauw, R. Panda, and I. N. Hajj. Early Probabilistic Noise Estimation for Capacitively Coupled Interconnects. IEEE Trans. on Computer-Aided Design of Integrated Circuits and Systems, 22, March 2003.

[5] M. Cho and D. Z. Pan. BoxRouter: A New Global Router Based on Box Expansion and Progressive ILP. In Proc. Design Automation Conf., July 2006.

[6] S. J. Fang, T. H. Smith, G. B. Shinn, J. A. Stefani, and D. S. Boning. Advanced Process Control in Dielectric Chemical Mechanical Polishing (CMP). In Chemical Mechanical Polish for ULSI Multilevel Interconnection Conference, Feb 1999.

[7] T. E. Gbondo-Tugbawa. Chip-Scale Modeling of Pattern Dependencies in Copper Chemical Mechanical Polishing Process. In Ph.D. Thesis, Massachusetts Institute of Technology, 2002.

[8] R. T. Hadsell and P. H. Madden. Improved Global Routing through Congestion Estimation. In Proc. Design Automation Conf., June 2003.

[9] L. He, A. B. Kahng, K. Tam, and J. Xiong. Design of IC Interconnects with Accurate Modeling of CMP. In Int. Society for Optical Engineering (SPIE) Symp. on Microlithograhpy, Mar 2005.

[10] T.-Y. Ho, Y.-W. Change, S.-J. Chen, and D. T. Lee. A Fast Crosstalk- and Performance-Driven Multilevel Routing System. In Proc. Int. Conf. on Computer Aided Design, Nov 2003.

[11] http://www.ece.ucsb.edu/ kastner/labyrinth/.

[12] http://www.praesagus.com/.

[13] J. Hu and S. Sapatnekar. A Timing-Constrained Algorithm for Simultaneous Global Routing of Multimple Nets. In Proc. Int. Conf. on Computer Aided Design, 2000.

[14] J. Hu and S. Sapatnekar. A Survey On Multi-net Global Routing for Integrated Circuits. Integration, the VLSI Journal, 31, 2002.

[15] S. Im, N. Srivastava, K. Banerjee, and K. E. Goodson. Scaling Analysis of Multilevel Interconnect Temperatrues for High-Performance ICs. IEEE Trans. on Electron Devices, 52, Dec 2005.

[16] International Technology Roadmap for Semiconductors (ITRS). 2005.

[17] R. Kastner, E. Bozorgzadeh, and M. Sarrafzadeh. Pattern Routing: Use and Theory for Increasing Predictability and Avoiding Coupling. IEEE Trans. on Computer-Aided
Design of Integrated Circuits and Systems, 21, July 2002.

[18] D. A. Kirkpatrick and A. L. Sangiovanni-Vincentelli. Techniques For Crosstalk Avoidance In The Physical Design Of High-performance Digital Systems. In Proc. Int. Conf. on Computer Aided Design, 1994.

[19] S. Lakshminarayanan, P. J. Wright, and J. Pallinti. Electrical characterization of the copper CMP process and derivation of metal layout rules. IEEE Trans. on Semiconductor Manufacturing, 16, Nov 2003.

[20] W.-S. Lee, K.-H. Lee, J.-K. Park, T.-K. Kim, and Y.-K. Park. Investigation of the capacitance deviation due to metal-fills and the effective interconnect geometry modeling. In Proc. Int. Symp. on Quality Electronic Design, Nov 2003.

[21] X. Qi, A. Gyure, Y. Luo, S. C. Lo, M. Shahram, and K. Singhal. Emerging technologies: Measurement and characterization of pattern dependent process variations of interconnect resistance, capacitance and inductance in nanometer technologies. In ACM Great Lakes symposium on VLSI, April 2006.

[22] P. Saxena and S. Gupta. On Integrating Power and Signal Routing for Shield Count Minimization in Congested Regions. IEEE Trans. on Computer-Aided Design of Integrated Circuits and Systems, 22, April 2003.

[23] R. Tian, D. F. Wong, and R. Boone. Model-Based Dummy Feature Placement for Oxide Chemical-Mechanical Polishing Manufacturability. IEEE Trans. on Computer-Aided Design of Integrated Circuits and Systems, 20, 2001.

[24] A. Vittal and M. Marek-Sadowska. Crosstalk reduction for VLSI. IEEE Trans. on Computer-Aided Design of Integrated Circuits and Systems, 16, March 1997.

[25] J. Westra, P. Groeneveld, T. Yan, and P. H. Madden. Global Routing: Metrics, Benchmarks, and Tools. In IEEE DATC Electronic Design Process, April 2005.

[26] D. Wu, J. Hu, and R. Mahapatra. Coupling Aware Timing Optimization and Antenna Avoidance in Layer Assignment. In Proc. Int. Symp. on Physical Design, April 2005.

[27] D. Wu, J. Hu, M. Zhao, and R. Mahapatra. Timing Driven Track Routing Considering Coupling Capacitance. In Proc. Asia and South Pacific Design Automation Conf., 2005.

[28] J. Xu, X. Hong, T. Jing, Y. Cai, and J. Gu. A Novel Timing-Driven Global Routing Algorithm Considering Coupling Effects for High Performance Circuit Design. In Proc. Asia and South Pacific Design Automation Conf., Jan 2003.

[29] P. Zarkesh-Ha, S. Lakshminarayann, K. Doniger, W. Loh, and P. Wright. Impact of Interconnect Pattern Density Information on a 90nm Technology ASIC Design Flow. In Proc. Int. Symp. on Quality Electronic Design, Nov 2003.

[30] H. Zhou and D. F. Wong. Global routing with crosstalk constraints. IEEE Trans. on Computer-Aided Design of Integrated Circuits and Systems, 18, Nov 1998. 